\title{
Design and Development of Patient Health Monitoring System Using IoT
}

\author{
${ }^{1}$ Sumit Bhardwaj, ${ }^{2}$ Gaurav Dawar, ${ }^{3}$ Pulkit Batra, ${ }^{4}$ Rishabh Rajput, ${ }^{5}$ Punit Gupta \\ 1, 2,3,4 Amity School of Engineering, Amity University, Uttar Pradesh \\ ${ }_{5}^{5}$ Jaypee University of Information Technology Solan, Himachal Pradesh, India \\ *Email: Sumitb460@gmail.com, punitg07@gmail.com
}

Received: 09 ${ }^{\text {th }}$ July 2018, Accepted: 14 ${ }^{\text {th }}$ August 2018, Published: 31 ${ }^{\text {st }}$ August 2018

\begin{abstract}
Observing your adored ones turns into a troublesome assignment in the cutting edge life. Monitoring the wellbeing status of the patient at home is a troublesome errand. Particularly old matured patients ought to be occasionally checked and their friends and family should be educated about their wellbeing status every once in a while at work. So we propose an imaginative framework that computerized this assignment easily. Our framework advances a brilliant patient wellbeing following framework that utilizations Sensors to track persistent wellbeing and utilizations web to advise their friends and family if there should arise an occurrence of any issues. Our framework utilizes temperature and in addition pulse detecting to monitor tolerant wellbeing. The sensors are associated with an arduino to track the status which is thus interfaced to a LCD show and in addition WiFi association so as to transmit alarms. On the off chance that framework identifies any sudden changes in quiet pulse or body temperature, the framework consequently alarms the client about the patient's status over IOT and furthermore indicates subtle elements of pulse and temperature of patient live finished the web. In this way IOT based patient wellbeing following framework adequately utilizes web to screen understanding wellbeing details and spare lives on time.
\end{abstract}

Keywords: Heartbeat Sensor, Humidity Sensor, Temperature Sensor, Galvanic Skin Response, Microcontroller, Concept, Wi-Fi Module.

\section{Introduction}

In this project, internet of things(IOT) based checking framework has been proposed to screen the patient naturally (more particularly associated sensors organize) that gathers the status data which incorporate patient's heart rate, body temperature, stickiness, surprising body development, galvanic skin reaction identifier and sends these information to the cloud by means of Wi-Fi modulator. Therefore, patient's specialist and attendants can see his present wellbeing condition regardless of where they are. If there should be an occurrence of crisis if the patient's wellbeing condition isn't steady (basic), the framework naturally sends a push notice utilizing a bell to specialists and medical caretakers. This would help the specialist to screen his patient from anyplace and in addition causes the patient's relatives to see his (understanding) wellbeing condition remotely (with restricted access).

IOT understanding checking has five sensors. Initially first is the heartrate detector, 2nd is temperature detector, the $3 \mathrm{rd}$ one is galvanic skin response sensor, the 4th one is flex sensor and the fifth one is the accelerometer sensor. The task is very valuable since the doctors can evaluate persistent parameters just by going by hotspot or URL. IOT applications are additionally being created. So the people who are close to patient can track the patient's wellbeing through the IOS or android applications. To work IOT based wellbeing checking framework venture, you require a Wi-Fi association. The Arduino board i.e. atmega 328 interfaces with the WiFi module. The undertaking will not work without a working Wi-Fi organize. You could make a Wi-Fi hotspot using a Wi-Fi module utilizing internet on your smart phone. The UNOatmega board persistently peruses contribution from these 5 detects. At that point it pushes the data to the hotspot by transmitting this information to an exact URL address. For instance in this undertaking, we have sent information after like clockwork.

This is a basic sensor based endeavor which has the latest advancement executed in it. Additionally, it has various applications and purposes of enthusiasm as indicated underneath.

1) IOT Health is one of the most asking for ground in the therapeutic zone. The errand is for, senior people in our home. Similarly for the senior national living alone or living with 1 or 2 people. This endeavor really shows steady when relatives need to go out for some emergency work.

2) IOT is also a very good thing for the people who can't go to the doctor on the daily basis such as disable patients. Incapacitate patients who find it to a great degree difficult to go to authorities on ordinary timetable or for those patients who require endless checking from the master.

As of late, the development of web is gigantic and has been additionally reached out to interfacing things 
through web. All gadgets are associated with each other with different brilliant advancements to make around the world pervasive system called Internet of Thigs (IoT). The advancement of advances, for example, IoT produces tremendous measure of information, prompts new time of data. Information produced by the IoT gadgets are utilized for investigation and basic leadership process.

\section{Evolution of Idea}

Sometimes monitoring the well being of the patient at home is a trouble undertaking as they are totally depended on you. In particular the case of senior citizen patients which are ought to be intermittently checked and their friends and family should be always updated about their wellbeing status every now and then while they are at work. So we came up with an idea of having a device that would help them to keep a track of it. Our framework advances a keen patient wellbeing following framework that utilizations Sensors to track persistent wellbeing and utilizations web to advise their friends and family if there should arise an occurrence of any issues.

\section{Working Methodology}

In this section we have proposed our designed system. IoT devices for health care consist like temperature sensor, blood pressure sensor, heart beat sensor and network connection. We have proposed a low cost IoT device for health care using ATMEGA 328.

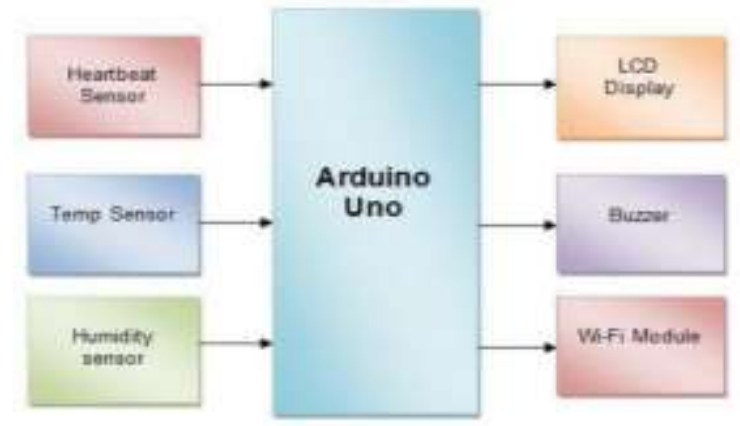

Fig 1 Basic Block Diagram

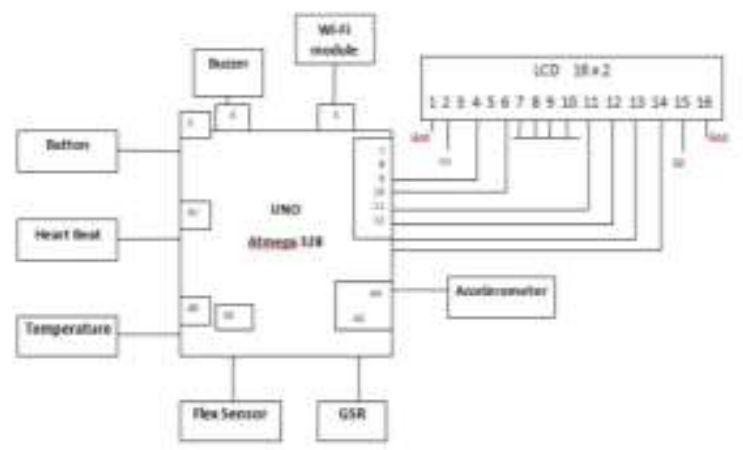

Fig 2 Basic Circuit Diagram

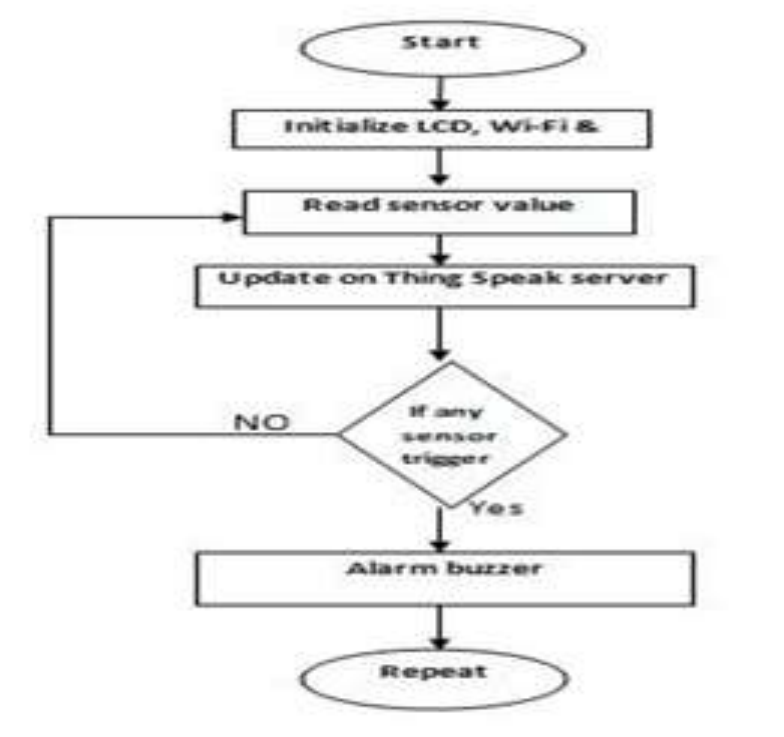

Fig 3 Flow Diagram

\section{Hardware Implementation}

This model architecture [10] uses RF which is transmitted and receive data wirelessly and arduino as main component of the model, this arduine is equipped with ATmega368 chip which have strek memory that can be read while compose. Each hardware is explained as follows:

Heartbeat Sensor: The pulse sensor relies upon the rule of photo phlethysmography. It gages the alteration in volume of blood through any organ of the body which causes a change in the light power through that organ (a vascular locale). In the event that there ought to emerge an event of employments where heart beat rate to be watched, the arranging of the pulsates is more essential.

LM35 Temperature Sensor: A generalized temperature sensor to sense the temperature reading in form of analog signal

Flex Sensor: The Flex Sensor protected innovation depends on resistive carbon components. As a variable printed resistor, the Flex Sensor accomplishes awesome shape factor on a thin adaptable substrate. At the point when the substrate is bowed, the sensor delivers a protection yield related to the twist spanthe littler the sweep, the higher the protection esteem. Accelerometer Sensor: The accelerometers measure the impacts of gravity and dynamic quickening, permitting assurance of a patient's introduction and developments. The accelerometer and ECG signals are contribution to a compact recorder and are sifted and digitized. Calculations were produced to consequently decide body position. The utilization of microfabricated accelerometers is a clinically doable strategy to decide body position and can be connected 
to future examinations corresponding body position with ECG or other physiologic information.

LCD: It is liquid crystal display, having flat panel comprising of two horizontal line, each line displaying sixteen character. It is easy programmable and low cost.

Wi-Fi Module: A module to connect the device wirelessly to the internet or intra network for communication using TCP/IP protocol.

GSR: A standout amongst the most touchy markers for passionate excitement is galvanic skin reaction (GSR), likewise alluded to as skin conductance (SC) or electro-dermal action (EDA). EDA balances the measure of sweat discharge from sweat organs. The measure of sweat organs differs over the human body. Buzzer: A standard to device to generate alert using fundamentals of electromagnetism.

Power Supply DC motor: it is powerful motor rotates 20 rotations per minute at $5 \mathrm{v}$ input.

\section{Software Implementation}

Arduino: A microcontroller board, contains on-board control supply, USB port to speak with PC, an Atmel microcontroller chip.

It disentangle the way toward making any control framework by giving the standard board that can be modified and associated with the framework without the need to any refined PCB outline and usage.

ThinkSpeak: It is the technology that is used in the project to demonstrate IOT. This is a cloud based open source platform provided to connect your device to upload and store the readings and can further be used to analyze filter and plot the data. It allows to deploy various categories of sensors to be deployed.

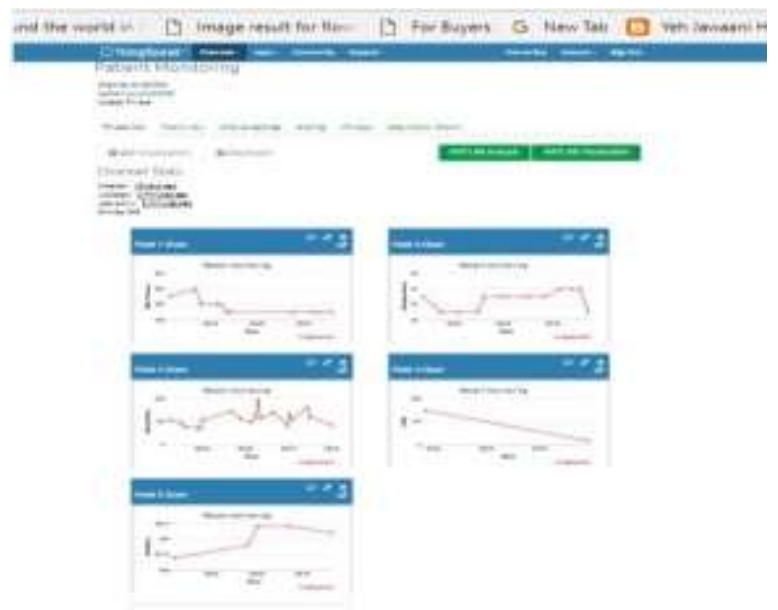

Fig 4 Graphical Representation of Data

\section{Conclusion}

From experimental result section, it is clear that proposed intelligent system is able to show and log the live system reading from remote health care kit. System uses a cloud system which is capable of connecting to $\mathrm{n}$ such devices and can deliver live reading at all time of all devices. The main idea of this system is to minimize the cost of system and gather more patients live health readings.

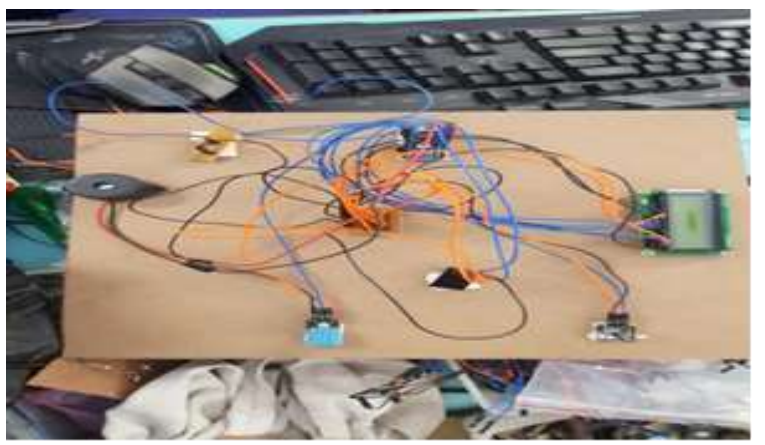

Fig 5 Circuit Diagram of Patient Health Monitoring System

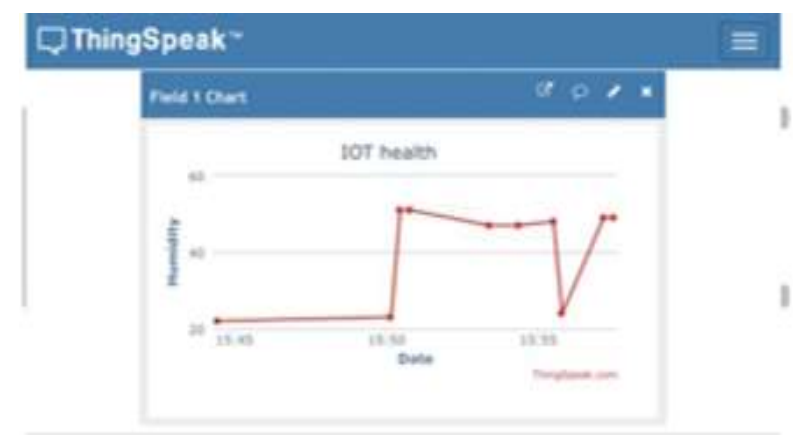

Fig 6 Graph for Humidity

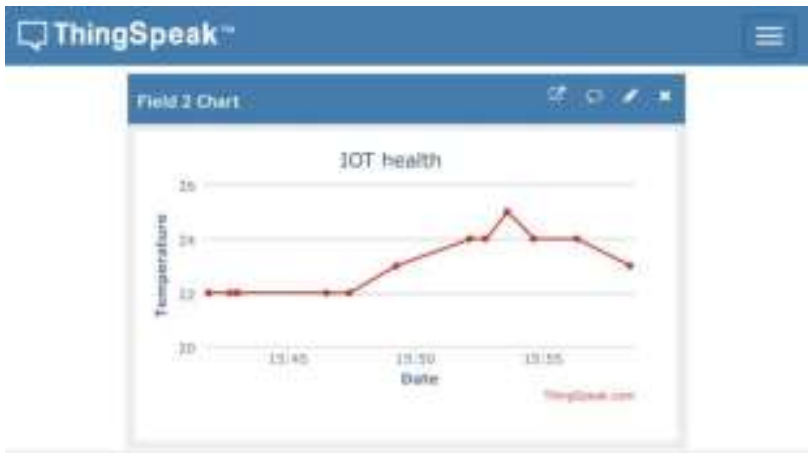

Fig 7 Graph for Temperature 


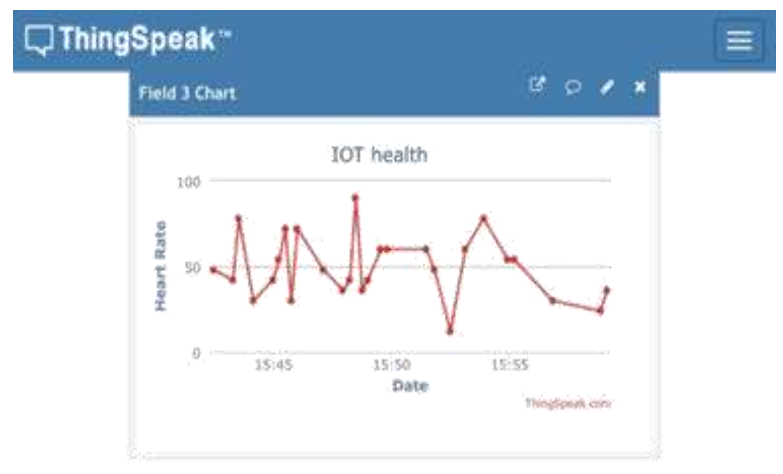

Fig 8 Graph for Heart Rate

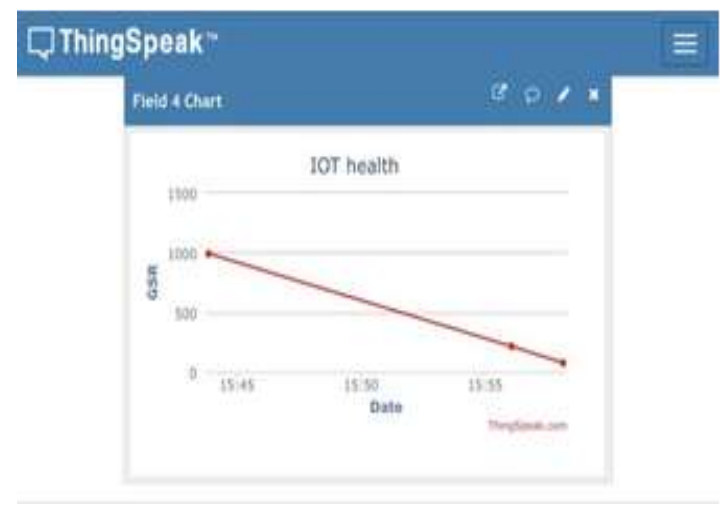

Fig 9 Graph for GSR

\section{References}

1. Whitmore, A. Agarwal, and L. Da Xu, The Internet of Things - A survey of topics and trends, Information Systems Frontiers, vol. 17, pp. 261-274 (2015)

2. S. Li, L. D. Xu, and S. Zhao, The internet of things: a survey, Information Systems Frontiers, vol. 17, pp. 243-259 (2015)

3. Bandana Mallick, Ajit Kumar Patro "heart rate monitoring system using fingertip through Arduino and processing software" International Journal of Science, Engineering and Technology Research (IJSETR), Volume 5, Issue 1, January 2016.

4. Souvik Das, "The Development of a Microcontroller Based Low Cost Heart Rate Counter for Health Care Systems" International Journal of Engineering Trends and TechnologyVolume4Issue2- 2013.

5. Luigi Atzori et al, "The Internet of Things: A survey”, Computer Networks, Vol.54, pp. 2787-2805, 2010.

6. Eleonora Borgia, "The Internet of Thigs vision: Key features, application and open issues", Computer Communication, Vol.54, pp. 131, 2014.
7. https://www.elprocus.com/wpcontent/uploads/20 14/07/heartbeat-sensor.jpg

8. Gennaro Tartarisco, Giovanni Baldus, Daniele Corda, Rossella Raso, Antonino Arnao, Marcello Ferro, Andrea Gaggioli, Giovanni Pioggia, "Personal Health System architecture for stress monitoring and support to clinical decisions", Computer Communications Vol.35, pp.1296-1305, 2012.

9. Franca Delmastro, "Pervasive communications in healthcare", Computer Communications Vol.35, pp.1284-1295,2012.

10. Tao Liu , Yoshio Inoue, Kyoko Shibata, "Development of a wearable sensor system for quantitative gait analysis", Measurement Vol. 42, pp.978-988, 2009.

11. Stefano Abbate, Marco Avvenuti, Francesco Bonatesta, Guglielmo Cola, Paolo Corsini, Alessio Vecchio, "A smartphone-based fall detection system", Pervasive and Mobile Computing Vol. 8,pp.883-899 , 2012.

12. Mohammed Riyadh Abdmeziem, Djamel Tandjaoui, "An end-to-end secure key management protocol for e-health applications", Computers and Electrical Engineering Vol.44, pp.184-197, 2015.

13. Eleonora Borgia, "The Internet of Things vision: Key features, applications and open issues", Computer Communications Vol .54 ,pp. 1-31, 2014.

14. Cristina Elena Turcua, Cornel Octavian Turcua, "Internet of Things as Key Enabler for Sustainable Healthcare Delivery", Procedia - Social and Behavioral Sciences Vol. 73, pp. 251 - 256, 2013 